\title{
Evaluation of Effect Mobility Node on Underwater Sensor Network Using VBVA Routing
}

\author{
Eka Purwa Laksana ${ }^{1}$, Riri Fitri Sari ${ }^{2}$ \\ \{eka.purwalaksana@budiluhur.ac.id ${ }^{1}$,riri@ui.ac.id $\left.{ }^{2}\right\}$ \\ Faculty of Engineering Universitas Budi Luhur, Jl. Raya Ciledug, Jakarta, Indonesia ${ }^{1}$, Department \\ Electro, Universitas Indonesia, Jakarta, Indonesia ${ }^{2}$
}

\begin{abstract}
We will show evaluation of performance Vector Based Void Avoidance (VBVA) routing for application mobility node on Underwater Sensor Network (UWSN). The research method is building the simulation using Network Simulator (NS-2.30) under operating system Linux Ubuntu. The installed NS-2.30 is added with Aqua-Sim module developed by Peng Xie. The final output from NS-2 will be visualized to graphic and table that will be analyzed using measurement metric packet delivery ratio, throughput, delay, and packet loss and energy consumption using AWK scripts with added with several modifications. The result of this simulation indicates that increasing number of nodes will result in loss of quality. One of them is decrease in packet delivery ratio. The decline was caused by that many packet collisions happen.
\end{abstract}

Keywords: UWSN, Routing, VBVA, Network Simulator, Mobility.

\section{Introduction}

Earth is a planet that is more than $71 \%$ of its surface is covered by ocean, most of which have not been investigated. As one way to find out the aquatic environment, is to use an Underwater Sensor Network (UWSN). UWSN has become a challenging research material for researchers. There is a difference between an UWSN with terrestrial sensor network.

One of the fundamental differences between UWSN with a terrestrial sensor network is a communication method. Terrestrial network using electromagnetic waves or radio waves to transmit data. While UWSN using acoustic signals.

The next difference is the underwater sensor nodes usually move due to water flow. Furthermore, when compared with terrestrial sensor node, the underwater node is usually much larger, more energy consuming, difficult to recharge and more expensive. The reason is more expensive causing placement in a wide area will not be solid. When nodes are placed with distance between nodes which has been determined, then the stream will change the distance between nodes. Because of the changing position of the nodes of the starting position, then the routing scheme will change. Changing the routing scheme will affect the success of the package is delivered. In this research, authors designed a simulation to measure the performance of routing for mobility nodes in UWSN using the NS-2. 


\section{Research Methodology}

\subsection{Underwater Sensor Network}

Underwater sensor network (UWSN) is a network that consists of multiple nodes that are placed in underwater with the aim to monitor the underwater environment. The greater characteristic of the UWSN the carrier wave using sound waves. UWSN is a new technique. This is significantly different from ground-based sensor networks. First, the Radio Frequency (RF) not function well in the underwater environment. In contrast, which acoustic signal should be used. Contrast to wireless link between ground-based sensors, underwater acoustic channel has large latency and low bandwidth.

Sound waves are waves which have the kind of pressure and caused by vibrations from an object in the transmission medium. Humans can only hear sound waves at a frequency between $20 \mathrm{~Hz}$ to $20 \mathrm{kHz}$. While the frequency used in the underwater sensor network is a frequency above $20 \mathrm{kHz}$ called ultrasonic.

In addition to sound waves, radio waves and optics had been tried applied to underwater sensor networks. However, experience high attenuation of radio waves in the sea water. Radio Frequency (RF) signal can propagate over long distances through the medium of water only at extra low frequencies $(30-300 \mathrm{~Hz}$ ), which require large antennae and high transmission power [1]. Therefore, the radio waves are not fit to use in underwater sensor networks.

For optical signals, the disadvantage is signal can propagate only short distances and Line Of Sight (LOS). The maximum transmission range of the optical wave is 8 meters in clear water (swimming pool). However, in highly turbid water with maximum visibility 1.4 meters, maximum range is $1 \mathrm{~m} \mathrm{[2].} \mathrm{Thus,} \mathrm{the} \mathrm{physical} \mathrm{communication} \mathrm{channel} \mathrm{which} \mathrm{most} \mathrm{appropriate}$ for the underwater sensor network is aqustic channel.

Second, most of the sensor nodes in the ground-based sensor networks usually static. In contrast, the majority of underwater sensor nodes except for some nodes will stay its place as it is equipped buoys move because of water flow. According to observations, underwater objects can move at speeds between 2-3 knots (3-6 km / h) [3]. Third, the period underwater sensor network system can be varied from a few minutes up to several years for a variety of applications ranging from short-term investigation of ships or for monitoring anti-submarine under water long term.

Speed of signal propagation in the underwater channel sensor networks is about $1.5 \mathrm{x} 103 \mathrm{~m} /$ $\mathrm{s}$, which is five times lower than the radio propagation speed $(3 \times 108 \mathrm{~m} / \mathrm{s})$. The bandwidth available for underwater sensor network is limited, depend on both transmission range and frequency. Frequency of underwater sensor networks is limited due to absorption, mostly underwater sensor network operating system below $30 \mathrm{kHz}$. [3].

\subsection{Vector-Based Void Avoidance (VBVA)}

Vector-Based Void Avoidance (VBVA) is the development of vector-based forwarding (VBF) with the capability to resolve the problem the void [4]. The node transmission in VBVA routing, which is as same as the VBF routing, can be defined by knowing the node location of its neighbor. The node will save the node forwarding location information as the node transmit the data packet. The forwading path of a packet is denoted as a vector and transmitted with a VBVA packet. When there is no void in the forwading path, VBVA will operate like VBF. What distinguishes between VBVA and VBF are VBVA is able to detect the presence of voids in the forwading path. by using the void avoidance mechanism, VBVA 
is able to find other forwading trajectories so that packet data transmission can still be done. As a result, network resistance can be improved.

Void node is defined as a node that has a vector value that is greater than the surrounding neighboring nodes. The trick is the node will listen to network and compare the value of the vector of its neighbor nodes. To overcome the void problems, VBVA uses two mechanisms such as vector-shift and back pressure.

1. vector-shift mechanisms

On VBVA if the node knows that there is a void node in the packet forwarding path, then that node will attempt to bypass the void by shifting the forwading vector [4]. To perform a shift vector, the void node will perform a broadcast packet control vector-shift to the surrounding nodes. When this packet control is received by the node, all nodes that are outside the void regional path will forward the forwading data packet by following the new path based on the new forwading vector from the sending node to the receiving node. This process is called vector-shift. When there is a node that can not find alternative routing paths although've done with the vector-shift mechanism, it must use other mechanisms that back pressure mechanism.

2. Back-pressure mechanism

When a node can not continue a data packet because it knows that it is the final node, it will broadcast a control packet called the BP packet (Back-Pressure) [4]. When the node receives the BP packet, each node will shift the forwading vector to get a new forwading path

\subsection{Reference Point Group Mobility (RPGM)}

Reference Point Group Mobility is a mobility model introduced by Xiaoyan Hong [3]. In this mobility model, each group has one central node. The central node which will be the reference of the location, speed, direction of all nodes within a group. Thus, the trajectory of the group specified by providing a way for the central node. Typically, nodes will be set uniformly in a geographic group. Each node is assigned a reference point for movement in a uniform group. Placement A node will be done randomly. The reference point scheme enables independent random movement behavior for each node, in addition to group movement..

\subsection{Aqua-Sim}

In the NS-2, the development of wireless packet CMU done for terrestrial wireless networks. As for the development of an underwater network should be added with aqua-sim [5], because the underwater environment different from the terrestrial environment. Specifically, under the water network has a long propagation delay and high damping. Terrestrial radio channel attenuation model cannot be applied again to lower the water network channels. In addition, the long propagation delay makes the collision behavior is very different from the behavior of collisions in radio networks that require different ways to simulate collisions in underwater sensor networks. In the NS-2, Aqua sim paralleled with wireless simulation package Carnegie Mellon University (CMU) as shown in Figure 1 [5]. 


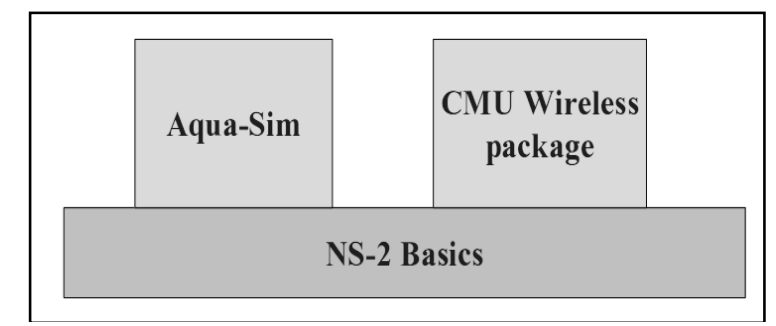

Fig. 1. The relationship between Aqua - Sim and other package of NS-2 [5].

\subsection{Aqua-Sim class diagram}

In Figure 2 Showing the class diagram of Aqua-Sim. "UnderwaterNode" is an abstraction of an underwater sensor node that contains various information from nodes such as location and speed of movement. "UnderwaterChannel" is an underwater acoustic channel where all packages will experience queues here before being sent. "UnderwaterChannel" also provides an interface for the top layer like the routing layer.

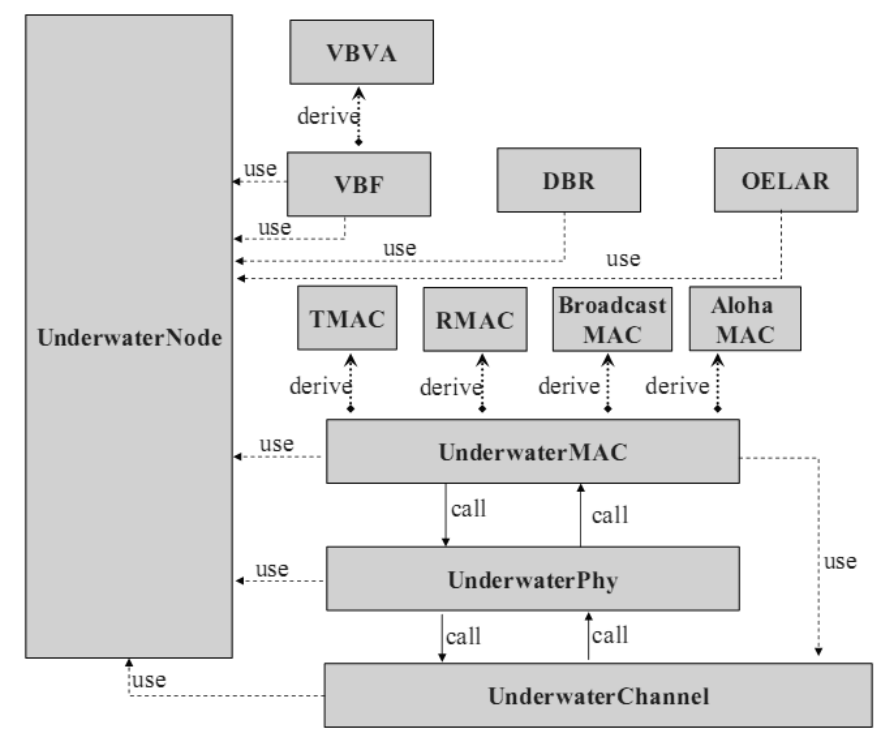

Fig. 2. Aqua class diagram - Sim [5].

\subsection{Network Simulator}

Network Simulator (NS) was first built in 1989 at the University of California Berkeley (UCB). In 1995 the Network Simulator-2 (NS-2) was first developed that is supported by the Defense Advanced Research Projects Agency (DARPA). NS-2 is a system that works on the operating system Unix/Linux.NS-2 can also run on a Windows operating system, but must use the Cygwin Linux environment it. Basically NS-2 is the OTcl interpreter with network 
simulation object libraries. The simulator supports a class hierarchy in $\mathrm{C}++$ (compiled hierarchy) and a similar class hierarchy in OTcl interpreter (interpreted hierarchy). The two hierarchies are interrelated to each other. Thisis the root of the hierarchy Object Tcl. Users create a simulator object (of class Simulator) recently through an interpreter, and reflected by an object is proportional to the compiled hierarchy. Once the Simulator object is created, then the methods to create the topology, nodes and other network components are called. NS-2 is also a simulator which triggered by an event (event-driven simulator). Scheduler runs by executing the next event which the most advance until finished, then re-run the next event. While communicating with the network passes the packet, but it does not spend a lot of time. If the components are made require a delay, then the event scheduler is used to publish events to the package and wait for stop event before the next event is run. Flowchart globally NS systems work can be seen in Figure 3.

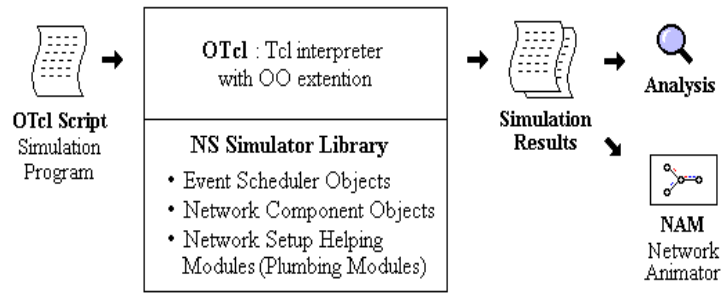

Fig. 3. Flowchart work system NS-2.

\subsection{Scenario}

Many of the techniques which can be used in evaluating a system. Simulation is one such technique. Simulation techniques are often used to test the proposed system in a short time and with low enough cost. For this reason, the simulator becomes the most important tool for researchers to researching new technologies. The author uses the NS-2 simulator to evaluate underwater sensor networks Because NS-2 has been used extensively and researchers have contributed to its development. This indicates that the simulator is quite complete and has many extensions for applications, protocols and traffic models different.

\subsubsection{Scenario simulation}

In analyzing the operating performance of the routing on the network UWSN, the authors conducted several scenarios of various traffic conditions with some variable parameters. The variable parameters in this simulation is the number of nodes and node speeds. The test is done by creating a test area with dimensions of $500 \mathrm{~m}$ X $500 \mathrm{~m}$ X $500 \mathrm{~m}$. In the testing area will be placed 10 to 50 nodes with 5 nodes each test differences. Distribution and movement of the nodes will be done randomly. Mobility of nodes will be moving at a speed of $0.8 \mathrm{~m} / \mathrm{s}$ and 1.6 $\mathrm{m} / \mathrm{s}$ (based on research) [3]. For each combination of variable parameter experiment is run ten times to obtain an average value. Scenarios to be run is used to see the effect of the routing operation VBVA to mobility node applications. This scenario also aims to obtain the effect of variations in the number of nodes and node speeds on packet delivery ratio, throughput, delay, and packet loss and energy consumption. There are two scenarios that will test them, that is:

1. Scenario 1: Traffic with a single source 
In the first scenario is one source node and destination node. Remaining nodes will serve as a relay node to be distributed randomly and perform random mobility. The relay nodes to function as an intermediary node sending packets from the source node to the destination node. The topology of the first scenario can be seen in figure 4 .

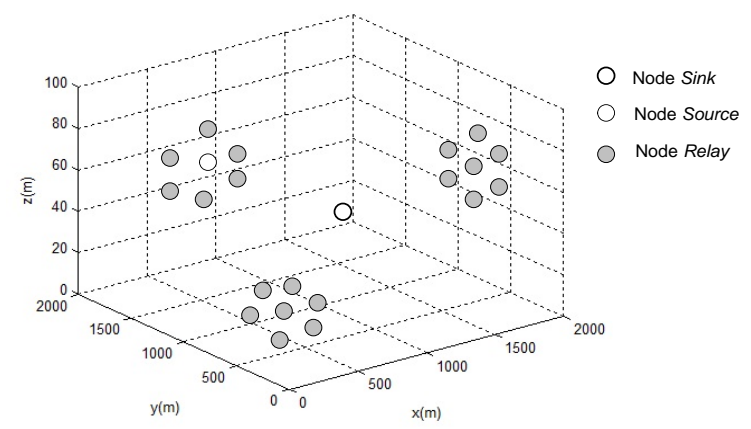

Fig. 4. Scenario 1.

2. Scenario 2: Traffic with multiple sources

In the second scenario there is one node that serves as the destination node and the remaining nodes will serve as a source node that can also function as a relay node. The topology of this second scenario can be seen in figure 5 .

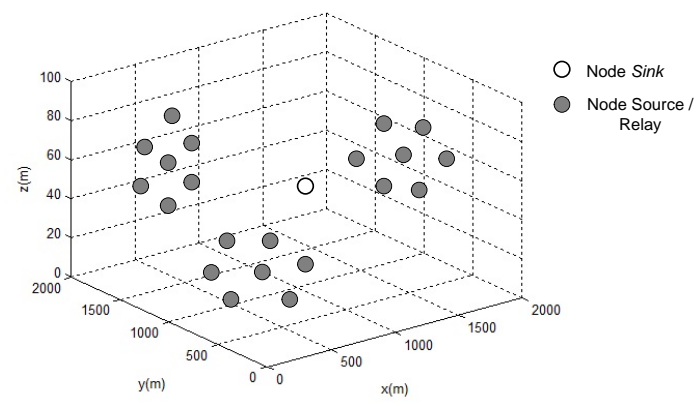

Fig. 5. Scenario 2.

\subsubsection{Simulation Parameters UWSN}

To get the results of a simulation of the system to be done, then it iis necessary to determine some parameters of the simulation. Simulation of routing in UWSN using the parameters shown in table 1.

Table 1. The Simulation Parameters.

\begin{tabular}{ll}
\hline Parameter & Value \\
\hline Bit rate & $10 \mathrm{Kbps}$ \\
\hline
\end{tabular}




\begin{tabular}{ll}
\hline frequency & $25 \mathrm{KHz}$ \\
Number of nodes & 50 \\
Delay & $25 \mu \mathrm{s}$ \\
Antenna & Omni directional \\
Tx power & $0,6 \mathrm{mWatt}$ \\
Rx power & $0,3 \mathrm{mWatt}$ \\
Node speed & $0,8 \mathrm{~m} / \mathrm{s}$ and $1,6 \mathrm{~m} / \mathrm{s}$ \\
\hline
\end{tabular}

\subsubsection{Performance Metrics}

1. Packet Delivery Ratio

Mathematically packet delivery ratio can be searched by the following equation:

2. Throughput

$$
\text { Packet delivery ratio(\%) }=\left(\frac{\Sigma \text { Packets received }}{\Sigma \text { sent Package }}\right) \times 100 \%
$$

Throughput can be calculated by dividing the number of data packets received by the time required for data transmission

3. Delay

$$
\text { Throughput }=\frac{\text { The size of received data }}{\text { The total time of data transmission }}
$$

Delay Time is the time required by a node to send data to another node during the simulation.

4. Packet loss

$$
\text { Delay }=\text { time packet received }- \text { the time the packet is sent }
$$

Packet loss can be determined by calculating the difference between the numbers of packets sent by the number of packets received. In the calculation of the number of packet loss will result in packet loss which is a representation of the number of packets lost or does not arrive at the destination node

\section{Energy consumption}

$$
\text { Packet Loss }=\frac{\text { Packages sent }- \text { received packets }}{\text { Packagessent }} \times 100 \%
$$

Energy consumption can be determined by calculating the difference between the initial energy given by the energy remaining after the node activity.

Energy consumption $=$ total initial energy - the number of residual energy

\section{Simulation and Analysis}

Figure 6 shows the packet delivery ratio, based on the traffic scenario with a single source node without movement, low node mobility and high node mobility. Overall, the packet delivery ratio will decrease as the density of nodes in the network. Increasing the number of nodes will gradually lead to loss of quality. One is a decrease in packet delivery ratio. The decline was caused by packet collision occurs more frequently. 


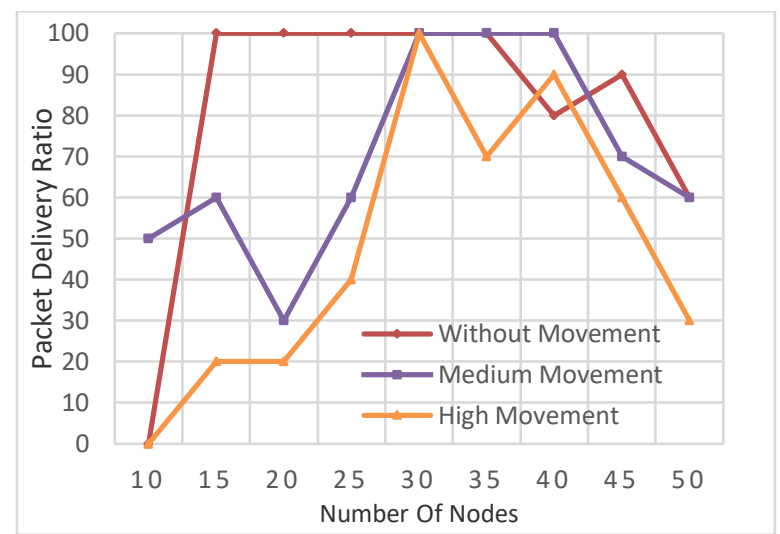

Fig. 6. Packet Delivery Ratio on traffic scenario with a single source.

Comparison of packet delivery ratio, based on the traffic scenario with multiple sources with no movement of nodes, nodes with moderate mobility and nodes with high mobility can be observed in Figure 7. In the traffic scenario with multiple sources, a decline in the success rate of packet delivery is gradually.

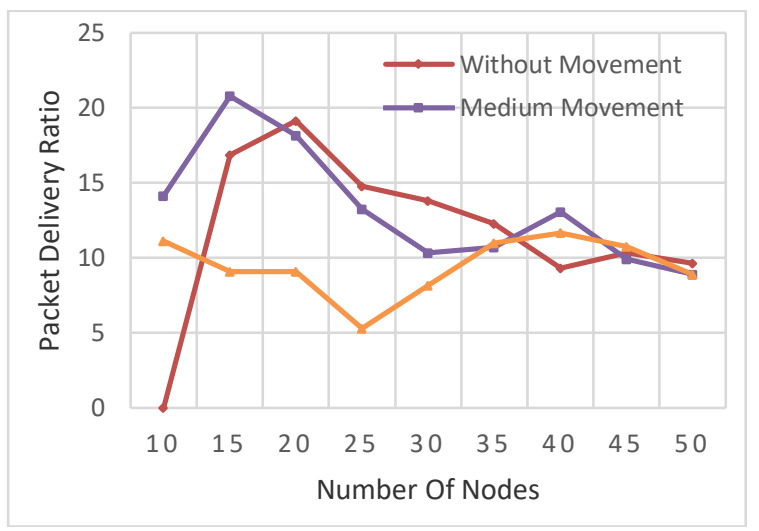

Fig. 7. Packet Delivery Ratio on traffic scenario with multiple sources.

Figure 8 shows the number of packet loss for traffic scenario with a single source node without movement, nodes with low mobility and nodes with high mobility. Packet loss is caused by several factors, including: the density of nodes in the network, the existence of voide nodes, thus causing packet drop. On the whole the more the number of active nodes in then the network packet loss will be even greater. 


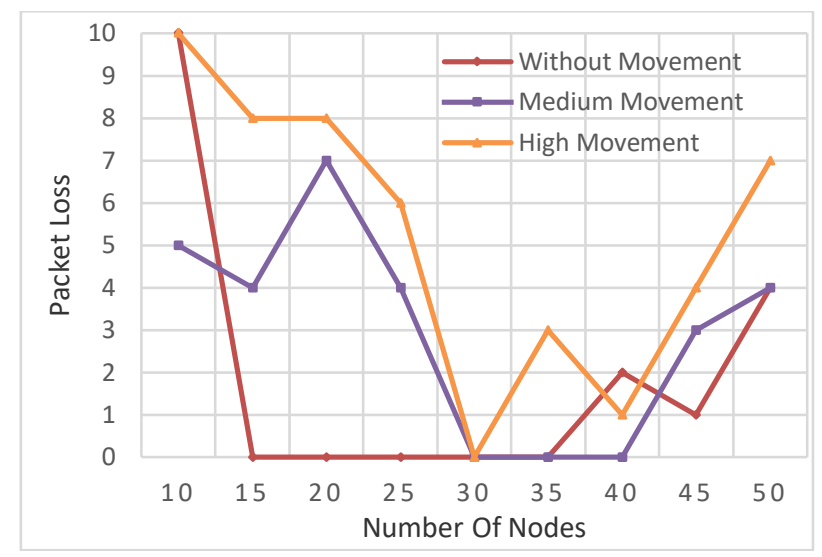

Fig. 8. Packet loss on traffic scenario with a single source.

Comparison of packet loss scenarios based on multiple sources of traffic can be seen in Figure 9. In the scenario of multiple sources of traffic increase in the number of packet loss occurs is gradually because of number of packets multiplied by the number of nodes minus 1 as the sink node which assigned only receive data, while the other served as a source node.

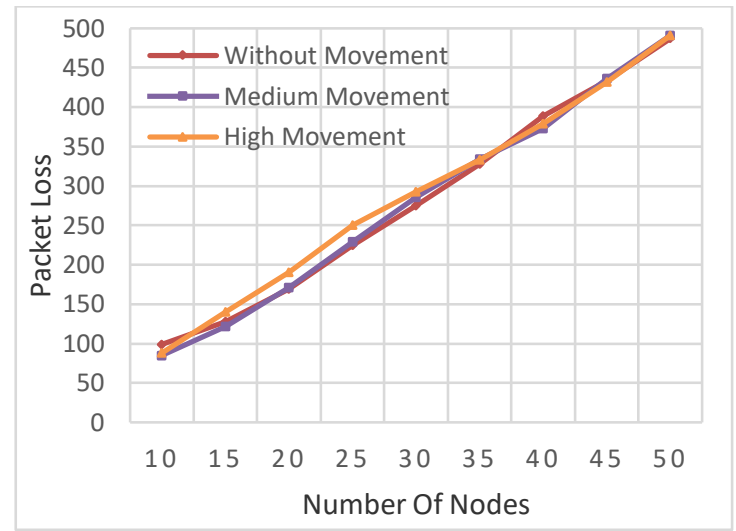

Fig. 9. Packet loss on traffic scenario with multiple sources.

Effect of node density on the throughput of network traffic based on a scenario with a single source is shown in Figure 10. Overall network throughput will increase with the increase in the number of active nodes. Network throughput for nodes without the movement of the node mobility better than low or high node mobility, because the increase is likely to be stable as the number of nodes in the network. 


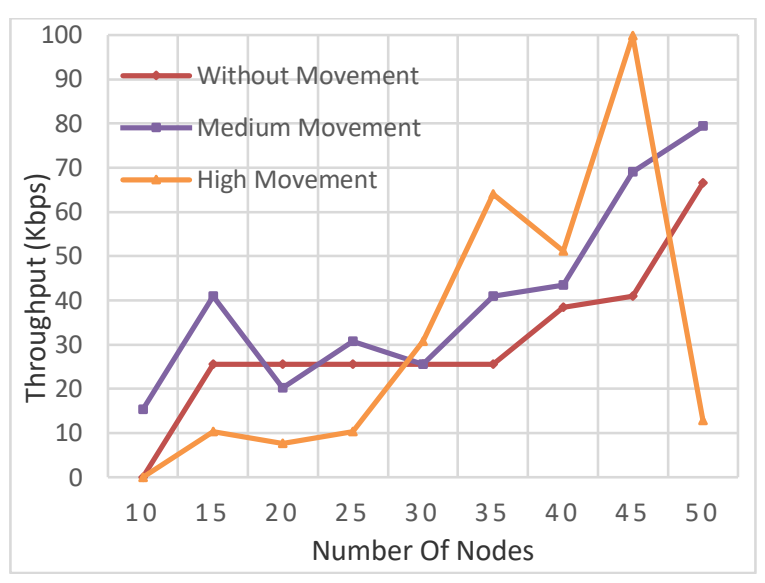

Fig. 10. Throughput on traffic scenario with a single source.

Figure 11 shows a comparison of the throughput of multiple sources of traffic scenarios. The increase in the number of nodes will have an impact on the throughput is increasingly rising.

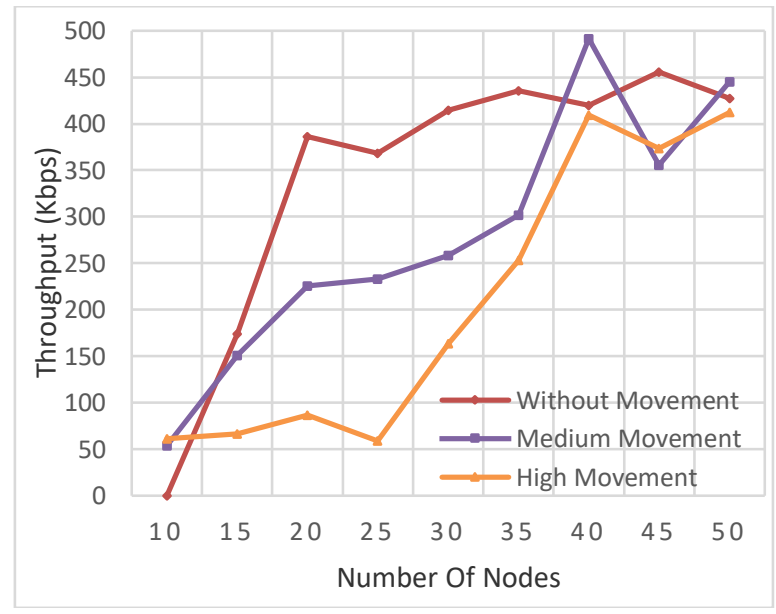

Fig. 11. Throughput on traffic scenario with multiple sources.

Delay is one of the parameters that determine the performance of a system. Significant differences between individual QoS parameters can be seen in the end-to-end delay. Delay in the network is more influenced by the existing traffic density due to the increasing number of nodes. From Figure 12 it can be seen that as the number of nodes in the network a little, delay nodes with high mobility and no movement of the lower nodes than the nodes with mobility being. 


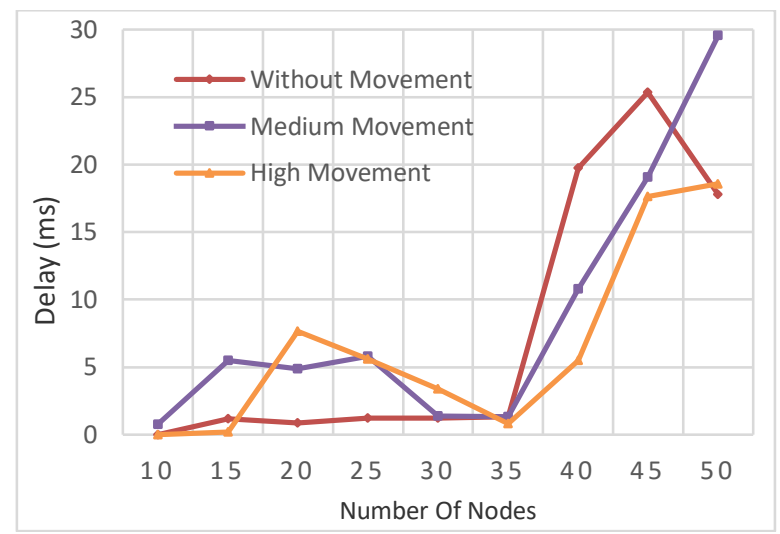

Fig. 12. Delay on traffic scenario with a single source.

Value of delay on the traffic scenario with multiple sources is shown in Figure 13. From the observations it can be seen that the average delay for nodes without movement when 10 nodes have the lowest delay value. However, when the node was 50, his highest delay.

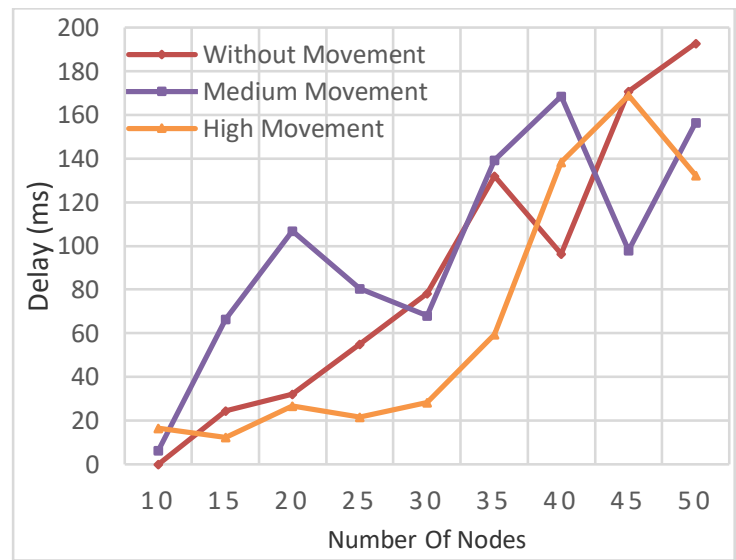

Fig. 13. Delay on traffic scenario with multiple sources.

Energy consumption is one of the parameters that determine the performance of a system. Energy consumption is important to note because power source for the underwater sensor network is difficult to recharge and more expensive. Energy consumption in the network is more influenced by the speed of the node mobility and number of nodes. From Figure 14 it can be seen that as the number of nodes in the network a little, the energy consumption of nodes with high mobility and no movement of nodes is lower than the mobility of nodes with mobility being. 


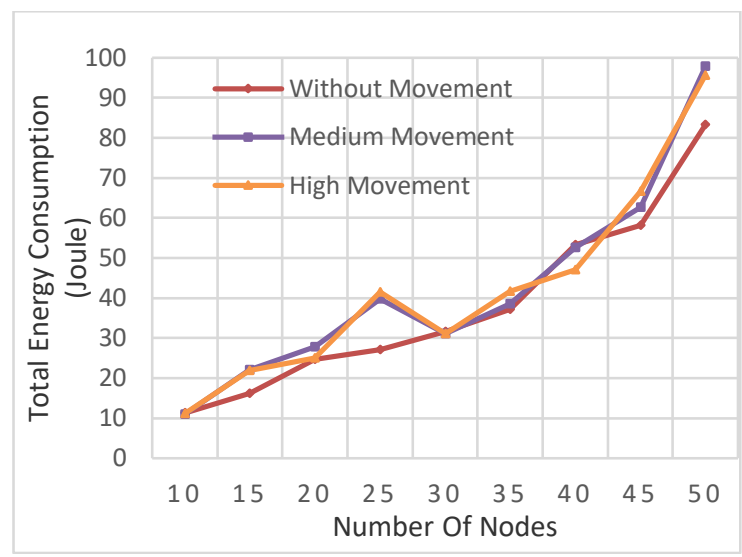

Fig. 14. Total Energy Consumption on traffic scenario with a single source.

Value of the energy consumption in the traffic scenario with multiple sources is shown in Figure 15. From the observations it can be seen that the energy consumption for nodes with high mobility requires less energy consumption compared to node without movement or low mobility.

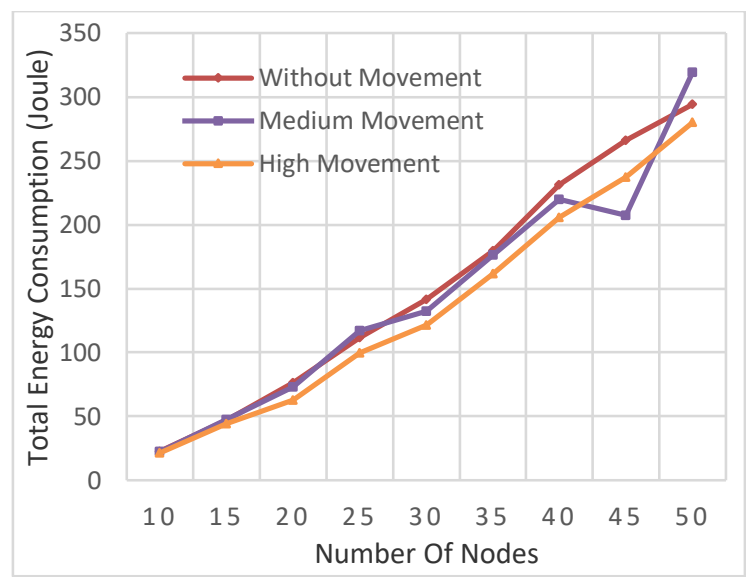

Fig. 15. Total Energy consumption on traffic scenario with multiple sources.

Based on the testing and analysis the scenario of a single source effective for 30 nodes. Because with using 30 nodes, for all mobility experiencing $100 \%$ packet delivery ratio. For the scenario to some sources, the effective number of nodes is 35 nodes, because when the number of nodes is more than 35 no significant changes its packet delivery ratio. Currently 35 nodes, nodes with no movement resulted in packet delivery ratio of $13.79 \%$, nodes with low mobility and $10.34 \%$ of nodes with high mobility of $8.15 \%$. 


\section{Conclusions}

Overall, the packet delivery ratio will decrease as the density of nodes in the network. Increasing the number of nodes will gradually lead to loss of quality. One is a decrease in packet delivery ratio. The decline was caused by packet collision occurs frequently.

Packet loss is caused by several factors, including: the density of nodes in the network, the existence of voide nodes, thus causing packet drop. On the whole, the number of active nodes, the packet loss network will be even greater.

In the scenario multiple sources, the traffic increase in the number of packet loss occurs is gradually because of number of packets multiplied by the number of nodes minus 1 as the sink node which assigned only receive data, while the other served as a source node.

Overall network throughput will increase with the increase in the number of active nodes. Network throughput for nodes without the movement of the node mobility better than the lowand high mobility of nodes, because the increase is likely to be stable as the number of nodes in the network.

Delay in the network is more influenced by the existing traffic density due to the increasing number of nodes. When the number of nodes in the network a little, delay nodes with high mobility and no movement of the lower nodes than the nodes with low mobility.

Energy consumption for nodes with high movement produces less energy consumption compared to node or nodes without any movement to the movement low.

\section{References}

[1]I. F. Akyildiz, D. Pompili, and T. Melodia, "State of the art in protocol research for underwater acoustic sensor networks,” ACM SIGMOBILE Mob. Comput. Commun. Rev.

[2]I. Vasilescu, K. Kotay, D. Rus, M. Dunbabin, and P. Corke, "Data collection, storage, and retrieval with an underwater sensor network," SenSys 2005 - Proc. 3rd Int. Conf. Embed. Networked Sens. Syst., pp. 154-165, 2005.

[3]Jun-Hong Cui; Jiejun Kong; M. Gerla; Shengli Zhou., "The challenges of building mobile underwater wireless networks for aquatic applications," IEEE Netw., vol. 20, no. 3, pp. 12-18, 2006.

[4]P. Xie, Z. Zhou, Z. Peng, J. H. Cui, and Z. Shi, "Void avoidance in Three-dimensional mobile underwater sensor networks," Lect. Notes Comput. Sci. (including Subser. Lect. Notes Artif. Intell. Lect. Notes Bioinformatics), vol. 5682 LNCS, pp. 305-314, 2009.

[5]P. X. P. Xie et al., "Aqua-Sim: An NS-2 based simulator for underwater sensor networks," Ocean. 2009, MTS/IEEE Biloxi - Mar. Technol. Our Futur. Glob. Local Challenges, pp. 1-7, 2009. 